\title{
ASPHALTIC MATERIAL IN THE CONTEXT OF GENERALIZED POROTHERMOELASTICITY
}

\author{
Mohammad H. Alawi \\ College of engineering and Islamic architecture, p.o.box:7398 Makkah, Saudi Arabia
}

\begin{abstract}
In this work, a mathematical model of generalized porothermoelasticity with one relaxation time for poroelastic half-space saturated with fluid will be constructed in the context of Youssef model (2007). We will obtain the general solution in the Laplace transform domain and apply it in a certain asphalt material which is thermally shocked on its bounding plane. The inversion of the Laplace transform will be obtained numerically and the numerical values of the temperature, stresses, strains and displacements will be illustrated graphically for the solid and the liquid.
\end{abstract}

\section{KEY-WORDS:}

Porothermoelasticity; asphaltic Material; Thermal shock.

\section{NOMENCLATURE}

$\begin{array}{ll}\mathrm{u}_{\mathrm{i}}, \mathrm{U}_{\mathrm{i}} & \text { The displacements of the skeleton and fluid phases } \\ \lambda, \mu, \mathrm{R}, \mathrm{Q} & \text { The poroelastic coefficients } \\ \mathrm{R}_{11}, \mathrm{R}_{12}, \mathrm{R}_{21}, \mathrm{R}_{22} \text { The mixed and thermal coefficients } \\ \theta^{\mathrm{s}}=\mathrm{T}^{\mathrm{s}}-\mathrm{T}_{0} & \text { The temperature increment of the solid where } \mathrm{T}^{\mathrm{s}} \text { is the solid } \\ \theta^{\mathrm{f}}=\mathrm{T}^{\mathrm{f}}-\mathrm{T}_{0} & \text { The temperature increment of the fluid where } \mathrm{T}^{\mathrm{f}} \text { is the fluid } \\ \mathrm{T}_{0} \text { The reference temperature } \\ \beta & \text { The porosity of the material } \\ \rho^{\mathrm{s}^{*}}, \rho^{\mathrm{f} *} & \text { The density of the solid and the liquid phases respectively } \\ \rho^{\mathrm{s}}=(1-\beta) \rho^{\mathrm{s}^{*}} & \text { The density of the solid phase per unit volume of bulk } \\ \rho^{\mathrm{f}}=\beta \rho^{\mathrm{f} *} & \text { The density of the solid phase per unit volume of bulk } \\ \rho_{11}=\rho^{\mathrm{s}}-\rho_{12} & \text { The mass coefficient of solid phase } \\ \rho_{22}=\rho^{\mathrm{f}}-\rho_{12} & \text { The mass coefficient of fluid phase } \\ \rho_{12} & \text { The dynamics coupling coefficient } \\ \mathrm{k}^{\mathrm{s}^{*}}, \mathrm{k}^{\mathrm{f} *} & \text { The thermal conductivity of the solid and the fluid phases } \\ \mathrm{k}^{\mathrm{s}}=(1-\beta) \mathrm{k}^{\mathrm{s}^{*}} & \text { The thermal conductivity of the solid phase } \\ \mathrm{k}^{\mathrm{f}}=\beta \mathrm{k}^{\mathrm{f} *} & \text { The thermal conductivity of the fluid phase } \\ \mathrm{k} & \text { The interface thermal conductivity }\end{array}$




\begin{tabular}{|c|c|}
\hline$\tau_{\mathrm{o}}^{\mathrm{s}}, \tau_{\mathrm{o}}^{\mathrm{f}}$ & The relaxation time of the solid and the fluid phases \\
\hline$\sigma_{\mathrm{ij}}$ & The stress components apply to the solid surface \\
\hline$\sigma$ & The normal stress apply to the fluid surface \\
\hline $\mathrm{e}_{\mathrm{ij}}$ & The strain component of the solid phase \\
\hline$\varepsilon$ & The strain component of the fluid phase \\
\hline$\alpha^{\mathrm{s}}, \alpha^{\mathrm{f}}$ & The coefficients of the thermal expansion of the phases \\
\hline$\alpha^{\mathrm{sf}}, \alpha^{\mathrm{fs}}$ & The thermoelastic couplings between the phases \\
\hline $\mathrm{C}_{\mathrm{E}}^{\mathrm{s}}, \mathrm{C}_{\mathrm{E}}^{\mathrm{f}}$ & The specific heat of the solid and the fluid phases \\
\hline $\mathrm{C}_{\mathrm{E}}^{\mathrm{sf}}$ & The specific heat couplings between the phases \\
\hline$\eta^{\mathrm{s}}=\frac{\rho^{\mathrm{s}} \mathrm{C}_{\mathrm{E}}^{\mathrm{s}}}{\mathrm{k}^{\mathrm{s}}}$ & The thermal viscosity of the solid \\
\hline$\eta^{\mathrm{f}}=\frac{\rho^{\mathrm{f}} \mathrm{C}_{\mathrm{E}}^{\mathrm{f}}}{\mathrm{k}^{\mathrm{f}}}$ & The thermal viscosity of the fluid \\
\hline$\eta=\frac{\rho_{12} C_{E}^{s f}}{k}$ & The thermal viscosity couplings between the phases \\
\hline $\mathrm{P}$ & $=3 \lambda+2 \mu$ \\
\hline $\mathrm{R}_{11}$ & $=\alpha^{\mathrm{s}} \mathrm{p}+\alpha^{\mathrm{fs}} \mathrm{Q}$ \\
\hline $\mathrm{R}_{22}$ & $=\alpha^{\mathrm{f}} \mathrm{R}+3 \alpha^{\mathrm{sf}} \mathrm{Q}$ \\
\hline $\mathrm{R}_{12}$ & $=\alpha^{\mathrm{f}} \mathrm{Q}+\alpha^{\mathrm{sf}} \mathrm{P}$ \\
\hline $\mathrm{F}_{11}$ & $=\rho^{\mathrm{s}} \mathrm{C}_{\mathrm{E}}^{\mathrm{s}}$ \\
\hline $\mathrm{F}_{22}$ & $=\rho^{\mathrm{f}} \mathrm{C}_{\mathrm{E}}^{\mathrm{f}}$ \\
\hline $\mathrm{F}_{12}$ & $=-\left(3 \alpha^{\mathrm{s}} \mathrm{R}_{12}+\alpha^{\mathrm{fs}} \mathrm{R}_{22}\right) \mathrm{T}_{\mathrm{o}}$ \\
\hline$F_{21}$ & $=-\left(3 \alpha^{\mathrm{sf}} \mathrm{R}_{11}+\alpha^{\mathrm{f}} \mathrm{R}_{21}\right) \mathrm{T}_{\mathrm{o}}$ \\
\hline
\end{tabular}

\section{INTRODUCTION}

Due to many applications in the fields of geophysics, plasma physics and related topics, an increasing attention is being devoted to the interaction between fluid such as water and thermo elastic solid, which is the domain of the theory of porothermoelasticity. The field of porothermoelasticity has a wide range of applications especially in studying the effect of using the waste materials on disintegration of asphalt concrete mixture.

Porous materials make their appearance in a wide variety of settings, natural and artificial and in diverse technological applications. As a consequence, a variety of problems arise while dealing with static and strength, fluid flow, heat conduction and the dynamics of such materials. In connection with the later, we note that problems of this kind are encountered in the prediction of behavior of sound-absorbing materials and in the area of exploration geophysics, the steadily growing literature bearing witness to the importance of the subject [1]. The problem of a fluidsaturated porous material has been studied for many years. A short list of papers pertinent to the present study includes Biot [2-3], Gassmann [4], Biot and Willis [5], Biot [6], Deresiewicz and Skalak [7], Mandl [8], Nur and Byerlee [9], Brown and Korringa [10], Rice and Cleary [11], Burridge and Keller [12], Zimmerman et al. [13-14], Berryman and Milton [15], Thompson and Willis [16], Pride et al. [17], Berryman and Wang [18], Tuncay and Corapcioglu [19], Alexander 
and Cheng [20], Charlez, P. A., and Heugas, O. [21], Abousleiman et al. [22], Ghassemi, A.[23] and Diek, A S. Tod [24].

The thermo-mechanical coupling in the poroelastic medium turns out to be of much greater complexity than that in the classical case of impermeable elastic solid. In addition to thermal and mechanical interaction within each phase, thermal and mechanical coupling occurs between the phases, thus, a mechanical or thermal change in one phase results in mechanical and thermal changes throughout the aggregate of asphaltic concrete mixtures. Following Biot, it takes one physical model to consist a homogeneous, isotropic, elastic matrix whose interstices are filled with a compressible ideal liquid both solid and liquid form continuous (and interacting) regions. While viscous stresses in the liquid are neglected, the liquid is assumed capable of exerting a velocity-dependent friction force on the skeleton. The mathematical model consists of two superposed continuous phases each separately filling the entire space occupied by the aggregate. Thus, there are two distinct elements at every point of space, each one characterized by its own displacement, stress, and temperature. During a thermo-mechanical process they may interact with a consequent exchange of momentum and energy.

Our development Proceeds by obtaining, the stress-strain-temperature relationships using the theory of the generalized thermo elasticity with one relaxation time "Lord-Shulman" [25]. Moreover, to the usual isobaric coefficients of thermal expansion of the single-phase materials, two coefficients appear which represent measures of each phase caused by temperature changes in the other phase.

As a result of the presence of these "coupling" coefficients, it follows that coefficient of thermal expansion of the dry material which differs than that of the saturated ones and the expansion of the liquid in the bulk is not the same as of the liquid phase. Putting into consideration the applications of geophysical interest, it takes the coefficient of proportionality in the dissipation term to be independent of frequency, that is, we confine ourselves to low-frequency motions. The last constituent of the theory is the equations of energy flux. Because the two phases in general, will be at different temperatures in each point of the material, there is a rise of a heat-source term in the energy equations representing the heat flux between the phases. It has been taken this "interphase heat transfer" to be proportional to the temperature difference between the phases. Finally, by using the uniqueness theorem the proof has been done.

Recently, Youssef has constructed a new version of theory of porothermoelasticity, using the modified Fourier law of heat conduction. The most important advantage for this theory, is predicting the finite speed of the wave propagation of the stress and the displacement as well as the heat [26].

In this paper, a mathematical model of generalized porothermoelasticity with one relaxation time for poroelastic half-space saturated with fluid will be constructed in the context of Youssef model. We will obtain the general solution in the Laplace transform domain and apply it in a certain asphalt material which is thermally shocked on its bounding plane. The inversion of the Laplace transform will be obtained numerically and the numerical values of the temperature, displacement and stress will be illustrated graphically.

\section{BASIC Formulations}

Starting by Youssef model of generalized porothermoelasticity [26], the linear governing equations of isotropic, generalized porothermoelasticity in absence of body forces and heat sources, are 
(i)- Equations of motion

$$
\begin{aligned}
& \mu u_{i, j j}+(\lambda+\mu) u_{j, i j}+Q U_{i, i i}-R_{11} \theta_{, i}^{s}-R_{12} \theta_{, i}^{f}=\rho_{11} \ddot{u}_{i}+\rho_{12} \ddot{U}_{i}, \\
& R U_{i, i i}+Q u_{j, i j}-R_{21} \theta_{, i}^{s}-R_{22} \theta_{, i}^{f}=\rho_{12} \ddot{u}_{i}+\rho_{22} \ddot{U}_{i} .
\end{aligned}
$$

(ii)- Heat equations

$$
\begin{aligned}
& \mathrm{k}^{\mathrm{s}} \theta_{, \mathrm{ii}}^{\mathrm{s}}=\left(\frac{\partial}{\partial \mathrm{t}}+\tau_{\mathrm{o}}^{\mathrm{s}} \frac{\partial^{2}}{\partial \mathrm{t}^{2}}\right)\left(\mathrm{F}_{11} \theta^{\mathrm{s}}+\mathrm{F}_{12} \theta^{\mathrm{f}}+\mathrm{T}_{\mathrm{o}} \mathrm{R}_{11} \mathrm{e}_{\mathrm{ii}}+\mathrm{T}_{\mathrm{o}} \mathrm{R}_{21} \varepsilon\right) \\
& \mathrm{k}^{\mathrm{f}} \theta_{, \mathrm{ii}}^{\mathrm{f}}=\left(\frac{\partial}{\partial \mathrm{t}}+\tau_{\mathrm{o}}^{\mathrm{f}} \frac{\partial^{2}}{\partial \mathrm{t}^{2}}\right)\left(\mathrm{F}_{21} \theta^{\mathrm{s}}+\mathrm{F}_{22} \theta^{\mathrm{f}}+\mathrm{T}_{\mathrm{o}} \mathrm{R}_{12} \mathrm{e}_{\mathrm{ii}}+\mathrm{T}_{\mathrm{o}} \mathrm{R}_{22} \varepsilon\right)
\end{aligned}
$$

(iii)- Constitutive equations

$$
\begin{aligned}
& \sigma_{\mathrm{ij}}=2 \mu \mathrm{e}_{\mathrm{ij}}+\lambda \mathrm{e}_{\mathrm{kk}} \delta_{\mathrm{ij}}+\left(\mathrm{Q} \varepsilon-\mathrm{R}_{11} \theta^{\mathrm{s}}-\mathrm{R}_{12} \theta^{\mathrm{f}}\right) \delta_{\mathrm{ij}}, \\
& \sigma=\mathrm{R} \varepsilon+\mathrm{Qe}_{\mathrm{kk}}-\mathrm{R}_{22} \theta^{\mathrm{f}}-\mathrm{R}_{21} \theta^{\mathrm{s}} . \\
& \mathrm{e}_{\mathrm{ij}}=\frac{1}{2}\left(\mathrm{u}_{\mathrm{i}, \mathrm{j}}+\mathrm{u}_{\mathrm{j}, \mathrm{i}}\right), \mathrm{e}_{\mathrm{ii}}=\mathrm{e}=\mathrm{u}_{\mathrm{i}, \mathrm{i}} \\
& \varepsilon=\mathrm{U}_{\mathrm{i}, \mathrm{i}} .
\end{aligned}
$$

\section{FORMULATION THE PROBLEM}

We will consider one dimensional half-space $0 \leq x<\infty$ is filled with porous, isotropic and elastic material which is considered to be at rest initially. The displacement will be considered to be in one dimensional as follows:

$$
\begin{aligned}
& \mathrm{u}_{1}=\mathrm{u}(\mathrm{x}, \mathrm{t}), \quad \mathrm{u}_{2}(\mathrm{x}, \mathrm{t})=\mathrm{u}_{3}(\mathrm{x}, \mathrm{t})=0 \\
& \mathrm{U}_{1}=\mathrm{U}(\mathrm{x}, \mathrm{t}), \quad \mathrm{U}_{2}(\mathrm{x}, \mathrm{t})=\mathrm{U}_{3}(\mathrm{x}, \mathrm{t})=0 .
\end{aligned}
$$

Then the governing equations (1)-(8) will take the forms:

(a) Equations of motion

$$
\begin{aligned}
& \frac{\partial^{2} \mathrm{u}}{\partial \mathrm{x}^{2}}+\frac{\mathrm{Q}}{(\lambda+2 \mu)} \frac{\partial^{2} \mathrm{U}}{\partial \mathrm{x}^{2}}-\frac{\mathrm{R}_{11}}{(\lambda+2 \mu)} \frac{\partial \theta^{\mathrm{s}}}{\partial \mathrm{x}}-\frac{\mathrm{R}_{12}}{(\lambda+2 \mu)} \frac{\partial \theta^{\mathrm{f}}}{\partial \mathrm{x}}=\frac{\rho_{11}}{(\lambda+2 \mu)} \ddot{\mathrm{u}}+\frac{\rho_{12}}{(\lambda+2 \mu)} \ddot{\mathrm{U}}, \\
& \frac{\partial^{2} \mathrm{U}}{\partial \mathrm{x}^{2}}+\frac{\mathrm{Q}}{\mathrm{R}} \frac{\partial^{2} \mathrm{u}}{\partial \mathrm{x}^{2}}-\frac{\mathrm{R}_{21}}{\mathrm{R}} \frac{\partial \theta^{\mathrm{s}}}{\partial \mathrm{x}}-\frac{\mathrm{R}_{22}}{\mathrm{R}} \frac{\partial \theta^{\mathrm{f}}}{\partial \mathrm{x}}=\frac{\rho_{21}}{\mathrm{R}} \ddot{\mathrm{u}}+\frac{\rho_{22}}{\mathrm{R}} \ddot{\mathrm{U}} .
\end{aligned}
$$

(b) Equation of heat

$$
\frac{\partial^{2} \theta^{s}}{\partial x^{2}}=\left(\frac{\partial}{\partial t}+\tau_{o}^{s} \frac{\partial^{2}}{\partial t^{2}}\right)\left(\frac{F_{11}}{k^{s}} \theta^{s}+\frac{F_{12}}{k^{s}} \theta^{f}+\frac{T_{0} R_{11}}{k^{s}} \frac{\partial u}{\partial x}+\frac{T_{0} R_{21}}{k^{s}} \frac{\partial U}{\partial x}\right),
$$




$$
\frac{\partial^{2} \theta^{\mathrm{f}}}{\partial \mathrm{x}^{2}}=\left(\frac{\partial}{\partial \mathrm{t}}+\tau_{\mathrm{o}}^{\mathrm{f}} \frac{\partial^{2}}{\partial \mathrm{t}^{2}}\right)\left(\frac{\mathrm{F}_{21}}{\mathrm{k}^{\mathrm{f}}} \theta^{\mathrm{s}}+\frac{\mathrm{F}_{22}}{\mathrm{k}^{\mathrm{f}}} \theta^{\mathrm{f}}+\frac{\mathrm{T}_{\mathrm{o}} \mathrm{R}_{12}}{\mathrm{k}^{\mathrm{f}}} \frac{\partial \mathrm{u}}{\partial \mathrm{x}}+\frac{\mathrm{T}_{\mathrm{o}} \mathrm{R}_{22}}{\mathrm{k}^{\mathrm{f}}} \frac{\partial \mathrm{U}}{\partial \mathrm{x}}\right) .
$$

(c) The constitutive relations

$$
\begin{aligned}
& \frac{\sigma_{x x}}{(\lambda+2 \mu)}=\frac{\partial u}{\partial x}+\frac{Q}{(\lambda+2 \mu)} \frac{\partial U}{\partial x}-\frac{R_{11}}{(\lambda+2 \mu)} \theta^{s}-\frac{R_{12}}{(\lambda+2 \mu)} \theta^{f} \\
& \frac{\sigma}{R}=\frac{\partial U}{\partial x}+\frac{Q}{R} \frac{\partial u}{\partial x}-\frac{R_{22}}{R} \theta^{f}-\frac{R_{21}}{R} \theta^{s} . \\
& e=\frac{\partial u}{\partial x} \\
& \varepsilon=\frac{\partial U}{\partial x} .
\end{aligned}
$$

Using the non-dimensional variables as follows:

$$
\left(u^{\prime}, U^{\prime}, x^{\prime}\right)=c_{o} \eta(u, U, x),\left(t^{\prime}, \tau_{0}^{\prime}\right)=c_{o}^{2} \eta\left(t, \tau_{0}\right),\left(\theta^{\prime s}, \theta^{\prime f}\right)=T_{0}\left(\theta^{s}, \theta^{f}\right), \sigma_{i j}^{\prime}=\frac{\sigma_{i j}}{\lambda+2 \mu}, \sigma^{\prime}=\frac{\sigma}{R}
$$

where

$$
\mathrm{c}_{\mathrm{o}}^{2}=\frac{\lambda+2 \mu}{\rho_{12}}, \eta=\frac{\rho_{12} \mathrm{C}_{\mathrm{E}}^{\mathrm{sf}}}{\mathrm{k}}
$$

Then, we get

$$
\begin{aligned}
& \frac{\partial^{2} \mathrm{u}}{\partial \mathrm{x}^{2}}+\frac{\mathrm{Q}}{(\lambda+2 \mu)} \frac{\partial^{2} \mathrm{U}}{\partial \mathrm{x}^{2}}-\frac{\mathrm{T}_{0} \mathrm{R}_{11}}{(\lambda+2 \mu)} \frac{\partial \theta^{\mathrm{s}}}{\partial \mathrm{x}}-\frac{\mathrm{T}_{0} \mathrm{R}_{12}}{(\lambda+2 \mu)} \frac{\partial \theta^{\mathrm{f}}}{\partial \mathrm{x}}=\frac{\rho_{11}}{\rho_{12}} \ddot{\mathrm{u}}+\ddot{\mathrm{U}}, \\
& \frac{\partial^{2} \mathrm{U}}{\partial \mathrm{x}^{2}}+\frac{\mathrm{Q}}{\mathrm{R}} \frac{\partial^{2} \mathrm{u}}{\partial \mathrm{x}^{2}}-\frac{\mathrm{T}_{0} \mathrm{R}_{21}}{\mathrm{R}} \frac{\partial \theta^{\mathrm{s}}}{\partial \mathrm{x}}-\frac{\mathrm{T}_{0} \mathrm{R}_{22}}{\mathrm{R}} \frac{\partial \theta^{\mathrm{f}}}{\partial \mathrm{x}}=\frac{(\lambda+2 \mu)}{\mathrm{R}} \ddot{\mathrm{u}}+\frac{\rho_{22}}{\rho_{12}} \frac{(\lambda+2 \mu)}{\mathrm{R}} \ddot{\mathrm{U}} . \\
& \frac{\partial^{2} \theta^{\mathrm{s}}}{\partial \mathrm{x}^{2}}=\left(\frac{\partial}{\partial \mathrm{t}}+\tau_{\mathrm{o}}^{\mathrm{s}} \frac{\partial^{2}}{\partial \mathrm{t}^{2}}\right)\left(\frac{\eta^{\mathrm{s}}}{\eta} \theta^{\mathrm{s}}+\frac{\mathrm{F}_{12}}{\mathrm{k}^{\mathrm{s}} \eta} \theta^{\mathrm{f}}+\frac{\mathrm{R}_{11}}{\mathrm{k}^{\mathrm{s}} \eta} \frac{\partial \mathrm{u}}{\partial \mathrm{x}}+\frac{\mathrm{R}_{21}}{\mathrm{k}^{\mathrm{s}} \eta} \frac{\partial \mathrm{U}}{\partial \mathrm{x}}\right), \\
& \frac{\partial^{2} \theta^{\mathrm{f}}}{\partial \mathrm{x}^{2}}=\left(\frac{\partial}{\partial \mathrm{t}}+\tau_{\mathrm{o}}^{\mathrm{f}} \frac{\partial^{2}}{\partial \mathrm{t}^{2}}\right)\left(\frac{\mathrm{F}_{21}}{\mathrm{k}^{\mathrm{f}} \eta} \theta^{\mathrm{s}}+\frac{\eta^{\mathrm{f}}}{\eta} \theta^{\mathrm{f}}+\frac{\mathrm{R}_{12}}{\mathrm{k}^{\mathrm{f}} \eta} \frac{\partial \mathrm{u}}{\partial \mathrm{x}}+\frac{\mathrm{R}_{22}}{\mathrm{k}^{\mathrm{f}} \eta} \frac{\partial \mathrm{U}}{\partial \mathrm{x}}\right) . \\
& \sigma_{x x}=\frac{\partial u}{\partial x}+\frac{Q}{(\lambda+2 \mu)} \frac{\partial U}{\partial x}-\frac{T_{0} R_{11}}{(\lambda+2 \mu)} \theta^{s}-\frac{T_{0} R_{12}}{(\lambda+2 \mu)} \theta^{\mathrm{f}} \\
& \sigma=\frac{\partial U}{\partial x}+\frac{Q}{R} \frac{\partial u}{\partial x}-\frac{T_{0} R_{22}}{R} \theta^{f}-\frac{T_{0} R_{21}}{R} \theta^{s} \text {. }
\end{aligned}
$$

In the above equation, we dropped the prime for convenient. 


\section{FORMULATION THE PROBLEM IN LAPLACE TRANSFORM DOMAIN}

Applying the Laplace transform for the both sides of the equations (19)-(24) which is defined as follows:

$$
\overline{\mathrm{f}}(\mathrm{s})=\int_{0}^{\infty} \mathrm{f}(\mathrm{t}) \mathrm{e}^{-\mathrm{st}} \mathrm{dt}
$$

then, we get

$$
\begin{aligned}
& \frac{d^{2} \bar{u}}{d x^{2}}=L_{11} \bar{u}+L_{12} \bar{U}+L_{13} \frac{d \bar{\theta}^{s}}{d x}+L_{14} \frac{d \bar{\theta}^{f}}{d x}, \\
& \frac{d^{2} \bar{U}}{d x^{2}}=L_{21} \bar{u}+L_{22} \bar{U}+L_{23} \frac{d \bar{\theta}^{s}}{d x}+L_{24} \frac{d \bar{\theta}^{f}}{d x}, \\
& \frac{d^{2} \bar{\theta}^{s}}{d x^{2}}=L_{31} \bar{\theta}^{s}+L_{32} \bar{\theta}^{f}+L_{33} \frac{d \bar{u}}{d x}+L_{34} \frac{d \bar{U}}{d x}, \\
& \frac{d^{2} \bar{\theta}^{f}}{d x^{2}}=L_{41} \bar{\theta}^{s}+L_{42} \bar{\theta}^{f}+L_{43} \frac{d \bar{u}}{d x}+L_{44} \frac{d \bar{U}}{d x}, \\
& \bar{\sigma} \overline{x x}_{x x}=\frac{d \bar{u}}{d x}+A \frac{d \bar{U}}{d x}-A_{11} \bar{\theta}^{s}-A_{12} \bar{\theta}^{f}, \\
& \bar{\sigma}=\frac{d \bar{U}}{d x}+B \frac{d \bar{u}}{d x}-A_{21} \bar{\theta}^{s}-A_{22} \bar{\theta}^{f}, \\
& \bar{e}=\frac{d \bar{u}}{d x} \\
& \bar{\varepsilon}=\frac{d \bar{U}}{d x} .
\end{aligned}
$$

where

$$
\begin{gathered}
\mathrm{L}_{11}=\left(\frac{\mathrm{C}_{11}-\mathrm{AC} \mathrm{C}_{21}}{1-\mathrm{AB}}\right), \mathrm{L}_{12}=\left(\frac{\mathrm{C}_{12}-\mathrm{AC}_{21}}{1-\mathrm{AB}}\right), \mathrm{L}_{13}=\left(\frac{\mathrm{A}_{11}-\mathrm{AA}_{21}}{1-\mathrm{AB}}\right), \mathrm{L}_{14}=\left(\frac{\mathrm{A}_{12}-\mathrm{AA}_{22}}{1-\mathrm{AB}}\right), \\
\mathrm{L}_{21}=\left(\frac{\mathrm{C}_{21}-\mathrm{BC}_{11}}{1-\mathrm{AB}}\right), \mathrm{L}_{22}=\left(\frac{\mathrm{C}_{22}-\mathrm{BC}_{12}}{1-\mathrm{AB}}\right), \mathrm{L}_{23}=\left(\frac{\mathrm{A}_{21}-\mathrm{BA}_{11}}{1-\mathrm{AB}}\right), \mathrm{L}_{24}=\left(\frac{\mathrm{A}_{22}-\mathrm{BA}_{12}}{1-\mathrm{AB}}\right), \\
\mathrm{L}_{31}=\frac{\left(\mathrm{s}+\tau_{\mathrm{o}}^{\mathrm{s}} \mathrm{s}^{2}\right) \eta^{\mathrm{s}}}{\eta}, \mathrm{L}_{32}=\frac{\left(\mathrm{s}+\tau_{\mathrm{o}}^{\mathrm{s}} \mathrm{s}^{2}\right) \mathrm{F}_{12}}{\mathrm{k}^{\mathrm{s}} \eta}, \mathrm{L}_{33}=\frac{\left(\mathrm{s}+\tau_{\mathrm{o}}^{\mathrm{s}} \mathrm{s}^{2}\right) \mathrm{R}_{11}}{\mathrm{k}^{\mathrm{s}} \eta}, \mathrm{L}_{34}=\frac{\left(\mathrm{s}+\tau_{\mathrm{o}}^{\mathrm{s}} \mathrm{s}^{2}\right) \mathrm{R}_{21}}{\mathrm{k}^{\mathrm{s}} \eta}, \\
\mathrm{L}_{41}=\frac{\left(\mathrm{s}+\tau_{\mathrm{o}}^{\mathrm{f}} \mathrm{s}^{2}\right) \mathrm{F}_{21}}{\mathrm{k}^{\mathrm{f}} \eta}, \mathrm{L}_{42}=\frac{\left(\mathrm{s}+\tau_{\mathrm{o}}^{\mathrm{f}} \mathrm{s}^{2}\right) \eta^{\mathrm{f}}}{\eta}, \mathrm{L}_{43}=\frac{\left(\mathrm{s}+\tau_{\mathrm{o}}^{\mathrm{f}} \mathrm{s}^{2}\right) \mathrm{R}_{12}}{\mathrm{k}^{\mathrm{f}} \eta}, \mathrm{L}_{44}=\frac{\left(\mathrm{s}+\tau_{\mathrm{o}}^{\mathrm{f}} \mathrm{s}^{2}\right) \mathrm{R}_{22}}{\mathrm{k}^{\mathrm{f}} \eta} . \\
\mathrm{A}=\frac{\mathrm{Q}}{(\lambda+2 \mu)}, \mathrm{A}_{11}=\frac{\mathrm{T}_{0} \mathrm{R}_{11}}{(\lambda+2 \mu)}, \mathrm{A}_{12}=\frac{\mathrm{T}_{0} \mathrm{R}_{12}}{(\lambda+2 \mu)}, \mathrm{C}_{11}=\frac{\rho_{11}}{\rho_{12}} \mathrm{~s}^{2}, \mathrm{C}_{12}=\mathrm{s}^{2} \\
\mathrm{~B}=\frac{\mathrm{Q}}{\mathrm{R}}, \mathrm{A}_{21}=\frac{\mathrm{T}_{0} \mathrm{R}_{21}}{\mathrm{R}}, \mathrm{A}_{22}=\frac{\mathrm{T}_{0} \mathrm{R}_{22}}{\mathrm{R}}, \mathrm{C}_{21}=\frac{(\lambda+2 \mu)}{\mathrm{R}} \mathrm{s}^{2}, \mathrm{C}_{22}=\frac{\rho_{22}}{\rho_{12}} \frac{(\lambda+2 \mu)}{\mathrm{R}} \mathrm{s}^{2},
\end{gathered}
$$

By using equations (25)-(28), we get 


$$
\begin{aligned}
& {\left[D_{\mathrm{x}}^{8}-\mathrm{aD}_{\mathrm{x}}^{6}+\mathrm{bD}_{\mathrm{x}}^{4}-\mathrm{cD}_{\mathrm{x}}^{2}+\mathrm{d}\right] \overline{\mathrm{u}}=0,} \\
& {\left[\mathrm{D}_{\mathrm{x}}^{8}-\mathrm{aD}_{\mathrm{x}}^{6}+\mathrm{bD}_{\mathrm{x}}^{4}-\mathrm{cD}_{\mathrm{x}}^{2}+\mathrm{d}\right] \overline{\mathrm{U}}=0,} \\
& {\left[\mathrm{D}_{\mathrm{x}}^{8}-\mathrm{aD}_{\mathrm{x}}^{6}+\mathrm{bD}_{\mathrm{x}}^{4}-\mathrm{cD}_{\mathrm{x}}^{2}+\mathrm{d}\right] \bar{\theta}^{\mathrm{s}}=0,} \\
& {\left[\mathrm{D}_{\mathrm{x}}^{8}-\mathrm{aD}_{\mathrm{x}}^{6}+\mathrm{bD}_{\mathrm{x}}^{4}-\mathrm{cD}_{\mathrm{x}}^{2}+\mathrm{d}\right] \bar{\theta}^{\mathrm{f}}=0,}
\end{aligned}
$$

where

$$
\begin{aligned}
\mathrm{a}= & \mathrm{L}_{11}+\mathrm{L}_{13} \mathrm{~L}_{33}+\mathrm{L}_{14} \mathrm{~L}_{43}+\mathrm{L}_{22}+\mathrm{L}_{23} \mathrm{~L}_{34}+\mathrm{L}_{24} \mathrm{~L}_{44}+\mathrm{L}_{31}+\mathrm{L}_{42} \\
\mathrm{~b}= & \mathrm{L}_{11}\left(\mathrm{~L}_{22}+\mathrm{L}_{23} \mathrm{~L}_{34}+\mathrm{L}_{24} \mathrm{~L}_{44}+\mathrm{L}_{31}+\mathrm{L}_{42}\right)-\mathrm{L}_{12}\left(\mathrm{~L}_{21}+\mathrm{L}_{23} \mathrm{~L}_{33}+\mathrm{L}_{24} \mathrm{~L}_{43}\right)- \\
& \mathrm{L}_{13}\left(\mathrm{~L}_{21} \mathrm{~L}_{34}-\mathrm{L}_{22} \mathrm{~L}_{33}+\mathrm{L}_{24}\left(\mathrm{~L}_{34} \mathrm{~L}_{43}-\mathrm{L}_{33} \mathrm{~L}_{44}\right)+\mathrm{L}_{32} \mathrm{~L}_{43}-\mathrm{L}_{33} \mathrm{~L}_{42}\right)- \\
& \mathrm{L}_{14}\left(\mathrm{~L}_{21} \mathrm{~L}_{44}-\mathrm{L}_{22} \mathrm{~L}_{43}+\mathrm{L}_{23}\left(\mathrm{~L}_{33} \mathrm{~L}_{44}-\mathrm{L}_{34} \mathrm{~L}_{43}\right)-\mathrm{L}_{31} \mathrm{~L}_{43}+\mathrm{L}_{33} \mathrm{~L}_{41}\right) \\
& +\mathrm{L}_{22}\left(\mathrm{~L}_{31}+\mathrm{L}_{42}\right)+\mathrm{L}_{23}\left(\mathrm{~L}_{34} \mathrm{~L}_{42}-\mathrm{L}_{32} \mathrm{~L}_{44}\right)+\mathrm{L}_{24}\left(\mathrm{~L}_{31} \mathrm{~L}_{44}-\mathrm{L}_{34} \mathrm{~L}_{41}\right)+\mathrm{L}_{31} \mathrm{~L}_{42}-\mathrm{L}_{32} \mathrm{~L}_{41} \\
\mathrm{c}= & \mathrm{L}_{11}\left(\mathrm{~L}_{22}\left(\mathrm{~L}_{31}+\mathrm{L}_{42}\right)+\mathrm{L}_{23}\left(\mathrm{~L}_{34} \mathrm{~L}_{42}-\mathrm{L}_{32} \mathrm{~L}_{44}\right)+\mathrm{L}_{24}\left(\mathrm{~L}_{31} \mathrm{~L}_{44}-\mathrm{L}_{34} \mathrm{~L}_{41}\right)+\mathrm{L}_{31} \mathrm{~L}_{42}-\mathrm{L}_{32} \mathrm{~L}_{41}\right) \\
& -\mathrm{L}_{12}\left(\mathrm{~L}_{21}\left(\mathrm{~L}_{31}+\mathrm{L}_{42}\right)+\mathrm{L}_{23}\left(\mathrm{~L}_{33} \mathrm{~L}_{42}-\mathrm{L}_{32} \mathrm{~L}_{43}\right)+\mathrm{L}_{24}\left(\mathrm{~L}_{31} \mathrm{~L}_{43}-\mathrm{L}_{33} \mathrm{~L}_{41}\right)\right) \\
& +\mathrm{L}_{13}\left(\mathrm{~L}_{21}\left(\mathrm{~L}_{32} \mathrm{~L}_{44}-\mathrm{L}_{34} \mathrm{~L}_{42}\right)+\mathrm{L}_{22}\left(\mathrm{~L}_{33} \mathrm{~L}_{42}-\mathrm{L}_{32} \mathrm{~L}_{43}\right)\right)-\mathrm{L}_{14}\left(\mathrm{~L}_{21}\left(\mathrm{~L}_{31} \mathrm{~L}_{44}-\mathrm{L}_{34} \mathrm{~L}_{41}\right)\right. \\
& \left.+\mathrm{L}_{22}\left(\mathrm{~L}_{33} \mathrm{~L}_{41}-\mathrm{L}_{31} \mathrm{~L}_{43}\right)\right)+\mathrm{L}_{22}\left(\mathrm{l}_{31} \mathrm{~L}_{42}-\mathrm{L}_{32} \mathrm{~L}_{41}\right) \\
\mathrm{d} & =\mathrm{L}_{11} \mathrm{~L}_{22}\left(\mathrm{~L}_{31} \mathrm{~L}_{42}-\mathrm{L}_{32} \mathrm{~L}_{41}\right)+\mathrm{L}_{12} \mathrm{~L}_{21}\left(\mathrm{~L}_{32} \mathrm{~L}_{41}-\mathrm{L}_{31} \mathrm{~L}_{42}\right) \\
\text { and } \quad & \mathrm{D}_{\mathrm{x}}^{\mathrm{n}}=\frac{\mathrm{d}^{\mathrm{n}}}{\mathrm{d} \mathrm{x}}
\end{aligned}
$$

According to equations (33)-(36) and to bounded state of functions at infinity, we can consider the following forms

$$
\begin{aligned}
& \overline{\mathrm{u}}(\mathrm{x}, \mathrm{s})=\sum_{\mathrm{i}=1}^{4} \alpha_{\mathrm{i}} \mathrm{e}^{-\lambda_{\mathrm{i}} \mathrm{x}}, \\
& \overline{\mathrm{U}}(\mathrm{x}, \mathrm{s})=\sum_{\mathrm{i}=1}^{4} \beta_{\mathrm{i}} \mathrm{e}^{-\lambda_{\mathrm{i}} \mathrm{x}}, \\
& \bar{\theta}^{\mathrm{s}}(\mathrm{x}, \mathrm{s})=\sum_{\mathrm{i}=1}^{4} \gamma_{\mathrm{i}} \mathrm{e}^{-\lambda_{\mathrm{i}} \mathrm{x}}, \\
& \bar{\theta}^{\mathrm{f}}(\mathrm{x}, \mathrm{s})=\sum_{\mathrm{i}=1}^{4} \omega_{\mathrm{i}} \mathrm{e}^{-\lambda_{\mathrm{i}} \mathrm{x}},
\end{aligned}
$$

where $\pm \lambda_{i}, i=1,2,3,4$ are the roots of the characteristic equation of the system (33)-(36) which takes the form

$$
\lambda^{8}-a \lambda^{6}+b \lambda^{4}-c \lambda^{2}+d=0
$$

To get the relations between the parameters $\beta_{i}, \gamma_{i}, \omega_{i}$ and $\alpha_{i}$, we will use equations (26)-(28) in the following forms 


$$
\begin{aligned}
& {\left[\mathrm{D}_{\mathrm{x}}^{2}-\mathrm{L}_{22}\right] \overline{\mathrm{U}}-\mathrm{L}_{23} \mathrm{D}_{\mathrm{x}} \bar{\theta}^{\mathrm{s}}-\mathrm{L}_{24} \mathrm{D}_{\mathrm{x}} \bar{\theta}^{\mathrm{f}}=\mathrm{L}_{21} \overline{\mathrm{u}},} \\
& -\mathrm{L}_{34} \overline{\mathrm{U}}+\left[\mathrm{D}_{\mathrm{x}}^{2}-\mathrm{L}_{31}\right] \bar{\theta}^{\mathrm{s}}-\mathrm{L}_{32} \bar{\theta}^{\mathrm{f}}=\mathrm{L}_{33} \mathrm{D}_{\mathrm{x}} \overline{\mathrm{u}}, \\
& -\mathrm{L}_{44} \mathrm{D}_{\mathrm{x}} \overline{\mathrm{U}}-\mathrm{L}_{41} \bar{\theta}^{\mathrm{s}}+\left[\mathrm{D}_{\mathrm{x}}^{2}-\mathrm{L}_{42}\right] \bar{\theta}^{\mathrm{f}}=\mathrm{L}_{43} \mathrm{D}_{\mathrm{x}} \overline{\mathrm{u}},
\end{aligned}
$$

Inserting the formulas in (37)-(40) into equations (42)-(44), we get

$$
\begin{aligned}
& \left(\lambda_{\mathrm{i}}^{2}-\mathrm{L}_{22}\right) \beta_{\mathrm{i}}+\mathrm{L}_{23} \lambda_{\mathrm{i}} \gamma_{\mathrm{i}}+\mathrm{L}_{24} \lambda_{\mathrm{i}} \omega_{\mathrm{i}}=\mathrm{L}_{21} \alpha_{\mathrm{i}}, \quad \mathrm{i}=1,2,3,4, \\
& -\mathrm{L}_{34} \beta_{\mathrm{i}}+\left(\lambda_{\mathrm{i}}^{2}-\mathrm{L}_{31}\right) \gamma_{\mathrm{i}}-\mathrm{L}_{32} \omega_{\mathrm{i}}=-\mathrm{L}_{33} \lambda_{\mathrm{i}} \alpha_{\mathrm{i}}, \quad \mathrm{i}=1,2,3,4, \\
& \mathrm{~L}_{44} \lambda_{\mathrm{i}} \beta_{\mathrm{i}}-\mathrm{L}_{41} \gamma_{\mathrm{i}}+\left(\lambda_{\mathrm{i}}^{2}-\mathrm{L}_{42}\right) \omega_{\mathrm{i}}=-\mathrm{L}_{43} \lambda_{\mathrm{i}} \alpha_{\mathrm{i}}, \quad \mathrm{i}=1,2,3,4,
\end{aligned}
$$

By solving the system in (45)-(47), we obtain

$$
\begin{aligned}
& \beta_{i}=\frac{H_{i}}{W_{i}} \alpha_{i}, i=1,2,3,4, \\
& \gamma_{i}=\frac{G_{i}}{W_{i}} \alpha_{i}, i=1,2,3,4, \\
& \gamma_{i}=\frac{F_{i}}{W_{i}} \alpha_{i}, i=1,2,3,4,
\end{aligned}
$$

Where

$$
\begin{aligned}
& \mathrm{H}_{\mathrm{i}}=-\left(\lambda_{\mathrm{i}}^{4}\left(\mathrm{~L}_{21}+\mathrm{L}_{23} \mathrm{~L}_{33}+\mathrm{L}_{24} \mathrm{~L}_{43}\right)-\lambda_{\mathrm{i}}^{2}\left(\mathrm{~L}_{21}\left(\mathrm{~L}_{31}+\mathrm{L}_{42}\right)+\mathrm{L}_{23}\left(\mathrm{~L}_{33} \mathrm{~L}_{42}-\mathrm{L}_{32} \mathrm{~L}_{43}\right)+\right.\right. \\
& \left.\left.\mathrm{L}_{24}\left(\mathrm{~L}_{31} \mathrm{~L}_{43}-\mathrm{L}_{33} \mathrm{~L}_{41}\right)\right)+\mathrm{L}_{21}\left(\mathrm{~L}_{31} \mathrm{~L}_{42}-\mathrm{L}_{32} \mathrm{~L}_{41}\right)\right), \mathrm{i}=1,2,3,4 \\
& \mathrm{G}_{\mathrm{i}}=\mathrm{L}_{33} \lambda_{\mathrm{i}}^{5}-\lambda_{\mathrm{i}}^{3}\left(\mathrm{~L}_{22} \mathrm{~L}_{33}+\mathrm{L}_{24} \mathrm{~L}_{33} \mathrm{~L}_{44}-\mathrm{L}_{32} \mathrm{~L}_{43}+\mathrm{L}_{33} \mathrm{~L}_{42}\right)-\mathrm{L}_{34} \lambda_{\mathrm{i}}^{2}\left(\mathrm{~L}_{21}+\mathrm{L}_{24} \mathrm{~L}_{43}\right) \\
& +\lambda\left(\mathrm{L}_{21} \mathrm{~L}_{32} \mathrm{~L}_{44}+\mathrm{L}_{22}\left(\mathrm{~L}_{33} \mathrm{~L}_{42}-\mathrm{L}_{32} \mathrm{~L}_{43}\right)\right)+\mathrm{L}_{21} \mathrm{~L}_{34} \mathrm{~L}_{42}, \mathrm{i}=1,2,3,4 \\
& \mathrm{~F}_{\mathrm{i}}=\mathrm{L}_{43} \lambda_{\mathrm{i}}^{5}+\lambda_{\mathrm{i}}^{3}\left(\mathrm{~L}_{21} \mathrm{~L}_{44}-\mathrm{L}_{22} \mathrm{~L}_{43}+\mathrm{L}_{23} \mathrm{~L}_{33} \mathrm{~L}_{44}-\mathrm{L}_{31} \mathrm{~L}_{43}+\mathrm{L}_{33} \mathrm{~L}_{41}\right)+\mathrm{L}_{23} \mathrm{~L}_{43} \mathrm{~L}_{34} \lambda_{\mathrm{i}}^{2} \\
& -\lambda_{\mathrm{i}}\left(\mathrm{L}_{21} \mathrm{~L}_{31} \mathrm{~L}_{44}+\mathrm{L}_{22}\left(\mathrm{~L}_{33} \mathrm{~L}_{41}-\mathrm{L}_{31} \mathrm{~L}_{43}\right)\right)-\mathrm{L}_{21} \mathrm{~L}_{34} \mathrm{~L}_{41}, \mathrm{i}=1,2,3,4 \\
& \mathrm{~W}_{\mathrm{i}}=-\lambda_{\mathrm{i}}^{6}+\lambda_{\mathrm{i}}^{4}\left(\mathrm{~L}_{22}+\mathrm{L}_{24} \mathrm{~L}_{44}+\mathrm{L}_{31}+\mathrm{L}_{42}\right)-\mathrm{L}_{23} \mathrm{~L}_{34} \lambda_{\mathrm{i}}^{3}-\lambda_{\mathrm{i}}^{2}\left(\mathrm{~L}_{22}\left(\mathrm{~L}_{31}+\mathrm{L}_{42}\right)-\mathrm{L}_{23} \mathrm{~L}_{32} \mathrm{~L}_{44}\right. \\
& \left.+\mathrm{L}_{24} \mathrm{~L}_{31} \mathrm{~L}_{44}+\mathrm{L}_{31} \mathrm{~L}_{42}-\mathrm{L}_{32} \mathrm{~L}_{41}\right)+\mathrm{L}_{34} \lambda_{\mathrm{i}}\left(\mathrm{L}_{23} \mathrm{~L}_{42}-\mathrm{L}_{24} \mathrm{~L}_{41}\right) \\
& +\mathrm{L}_{22}\left(\mathrm{~L}_{31} \mathrm{~L}_{42}-\mathrm{L}_{32} \mathrm{~L}_{41}\right), \mathrm{i}=1,2,3,4
\end{aligned}
$$

Hence, we have

$$
\begin{aligned}
& \overline{\mathrm{U}}(\mathrm{x}, \mathrm{s})=\sum_{\mathrm{i}=1}^{4} \frac{\mathrm{H}_{\mathrm{i}}}{\mathrm{W}_{\mathrm{i}}} \alpha_{\mathrm{i}} \mathrm{e}^{-\lambda_{\mathrm{i}} \mathrm{x}}, \\
& \bar{\theta}^{\mathrm{s}}(\mathrm{x}, \mathrm{s})=\sum_{\mathrm{i}=1}^{4} \frac{\mathrm{G}_{\mathrm{i}}}{\mathrm{W}_{\mathrm{i}}} \alpha_{\mathrm{i}} \mathrm{e}^{-\lambda_{\mathrm{i}} \mathrm{x}}, \\
& \bar{\theta}^{\mathrm{f}}(\mathrm{x}, \mathrm{s})=\sum_{\mathrm{i}=1}^{4} \frac{\mathrm{F}_{\mathrm{i}}}{\mathrm{W}_{\mathrm{i}}} \alpha_{\mathrm{i}} \mathrm{e}^{-\lambda_{\mathrm{i}} \mathrm{x}},
\end{aligned}
$$

To get the values of the parameters $\alpha_{i}$, we have to apply the boundary conditions as follows; 
(1) The thermal conditions

We will consider the bounding plane surface of the medium at $\mathrm{x}=0$ has been thermally loaded by thermal shock as follows:

$$
\theta^{\mathrm{s}}(0, \mathrm{t})=(1-\beta) \theta_{0} \mathrm{H}(\mathrm{t})
$$

and

$$
\theta^{\mathrm{f}}(0, \mathrm{t})=\beta \theta_{0} \mathrm{H}(\mathrm{t}), \quad(52)
$$

where $H(t)$ is the Heaviside unite step function and $\theta_{0}$ is constant which gives after using the Laplace transform the following conditions

$$
\bar{\theta}^{s}(0, s)=\frac{(1-\beta) \theta_{0}}{s},
$$

and

$$
\bar{\theta}^{\mathrm{f}}(0, \mathrm{~s})=\frac{\beta \theta_{0}}{\mathrm{~s}},
$$

(2) The mechanical conditions

We will consider the bounding plane surface of the medium at $x=0$ has been connected to a rigid surface which prevents any displacement to accrue on that surface, i.e.

$$
\mathrm{u}(0, \mathrm{t})=0 \text {, }
$$

and

$$
\mathrm{U}(0, \mathrm{t})=0
$$

which gives after using the Laplace transform the following conditions

$$
\overline{\mathrm{u}}(0, \mathrm{~s})=0,
$$

and

$$
\overline{\mathrm{U}}(0, \mathrm{~s})=0 \text {. }
$$

After using the boundary conditions in (53), (54), (57) and (58), we get the following system

$$
\begin{aligned}
& \sum_{i=1}^{4} \alpha_{i}=0, \\
& \sum_{i=1}^{4} \frac{\mathrm{H}_{\mathrm{i}}}{\mathrm{W}_{\mathrm{i}}} \alpha_{\mathrm{i}}=0, \\
& \sum_{\mathrm{i}=1}^{4} \frac{\mathrm{G}_{\mathrm{i}}}{\mathrm{W}_{\mathrm{i}}} \alpha_{\mathrm{i}}=\frac{(1-\beta) \theta_{0}}{\mathrm{~s}}, \\
& \sum_{\mathrm{i}=1}^{4} \frac{\mathrm{F}_{\mathrm{i}}}{\mathrm{W}_{\mathrm{i}}} \alpha_{\mathrm{i}}=\frac{\beta \theta_{0}}{\mathrm{~s}},
\end{aligned}
$$

Then we get 


$$
\begin{aligned}
& \alpha_{1}=\frac{\theta_{0} \mathrm{~W}_{1}}{\mathrm{~s} \Delta}\left[\begin{array}{l}
\mathrm{W}_{2}\left(\beta\left(\mathrm{F}_{3} \mathrm{H}_{4}-\mathrm{F}_{4} \mathrm{H}_{3}+\mathrm{G}_{3} \mathrm{H}_{4}-\mathrm{G}_{4} \mathrm{H}_{3}\right)-\mathrm{F}_{3} \mathrm{H}_{4}+\mathrm{F}_{4} \mathrm{H}_{3}\right)- \\
\mathrm{W}_{3}\left(\beta\left(\mathrm{F}_{2} \mathrm{H}_{4}-\mathrm{F}_{4} \mathrm{H}_{2}+\mathrm{G}_{2} \mathrm{H}_{4}-\mathrm{G}_{4} \mathrm{H}_{2}\right)-\mathrm{F}_{2} \mathrm{H}_{4}+\mathrm{F}_{4} \mathrm{H}_{2}\right)+ \\
\mathrm{W}_{4}\left(\beta\left(\mathrm{F}_{2} \mathrm{H}_{3}-\mathrm{F}_{3} \mathrm{H}_{2}+\mathrm{G}_{2} \mathrm{H}_{3}-\mathrm{G}_{3} \mathrm{H}_{2}\right)-\mathrm{F}_{2} \mathrm{H}_{3}+\mathrm{F}_{3} \mathrm{H}_{2}\right)
\end{array}\right], \\
& \alpha_{2}=-\frac{\theta_{0} \mathrm{~W}_{2}}{\mathrm{~s} \Delta}\left[\begin{array}{l}
\mathrm{W}_{1}\left(\beta\left(\mathrm{F}_{3} \mathrm{H}_{4}-\mathrm{F}_{4} \mathrm{H}_{3}+\mathrm{G}_{3} \mathrm{H}_{4}-\mathrm{G}_{4} \mathrm{H}_{3}\right)-\mathrm{F}_{3} \mathrm{H}_{4}+\mathrm{F}_{4} \mathrm{H}_{3}\right)- \\
\mathrm{W}_{3}\left(\beta\left(\mathrm{F}_{1} \mathrm{H}_{4}-\mathrm{F}_{4} \mathrm{H}_{1}+\mathrm{G}_{1} \mathrm{H}_{4}-\mathrm{G}_{4} \mathrm{H}_{1}\right)-\mathrm{F}_{1} \mathrm{H}_{4}+\mathrm{F}_{4} \mathrm{H}_{1}\right)+ \\
\mathrm{W}_{4}\left(\beta\left(\mathrm{F}_{1} \mathrm{H}_{3}-\mathrm{F}_{3} \mathrm{H}_{1}+\mathrm{G}_{1} \mathrm{H}_{3}-\mathrm{G}_{3} \mathrm{H}_{1}\right)-\mathrm{F}_{1} \mathrm{H}_{3}+\mathrm{F}_{3} \mathrm{H}_{1}\right)
\end{array}\right], \\
& \alpha_{3}=\frac{\theta_{0} \mathrm{~W}_{3}}{\mathrm{~s} \Delta}\left[\begin{array}{l}
\mathrm{W}_{1}\left(\beta\left(\mathrm{F}_{2} \mathrm{H}_{4}-\mathrm{F}_{4} \mathrm{H}_{2}+\mathrm{G}_{2} \mathrm{H}_{4}-\mathrm{G}_{4} \mathrm{H}_{2}\right)-\mathrm{F}_{2} \mathrm{H}_{4}+\mathrm{F}_{4} \mathrm{H}_{2}\right)- \\
\mathrm{W}_{2}\left(\beta\left(\mathrm{F}_{1} \mathrm{H}_{4}-\mathrm{F}_{4} \mathrm{H}_{1}+\mathrm{G}_{1} \mathrm{H}_{4}-\mathrm{G}_{4} \mathrm{H}_{1}\right)-\mathrm{F}_{1} \mathrm{H}_{4}+\mathrm{F}_{4} \mathrm{H}_{1}\right)+ \\
\mathrm{W}_{4}\left(\beta\left(\mathrm{F}_{1} \mathrm{H}_{2}-\mathrm{F}_{2} \mathrm{H}_{1}+\mathrm{G}_{1} \mathrm{H}_{2}-\mathrm{G}_{2} \mathrm{H}_{1}\right)-\mathrm{F}_{1} \mathrm{H}_{2}+\mathrm{F}_{2} \mathrm{H}_{1}\right)
\end{array}\right], \\
& \alpha_{4}=-\frac{\theta_{0} \mathrm{~W}_{4}}{\mathrm{~s} \Delta}\left[\begin{array}{l}
\mathrm{W}_{1}\left(\beta\left(\mathrm{F}_{2} \mathrm{H}_{3}-\mathrm{F}_{3} \mathrm{H}_{2}+\mathrm{G}_{2} \mathrm{H}_{3}-\mathrm{G}_{3} \mathrm{H}_{2}\right)-\mathrm{F}_{2} \mathrm{H}_{3}+\mathrm{F}_{3} \mathrm{H}_{2}\right)- \\
\mathrm{W}_{2}\left(\beta\left(\mathrm{F}_{1} \mathrm{H}_{3}-\mathrm{F}_{3} \mathrm{H}_{1}+\mathrm{G}_{1} \mathrm{H}_{3}-\mathrm{G}_{3} \mathrm{H}_{1}\right)-\mathrm{F}_{1} \mathrm{H}_{3}+\mathrm{F}_{3} \mathrm{H}_{1}\right)+ \\
\mathrm{W}_{3}\left(\beta\left(\mathrm{F}_{1} \mathrm{H}_{2}-\mathrm{F}_{2} \mathrm{H}_{1}+\mathrm{G}_{1} \mathrm{H}_{2}-\mathrm{G}_{2} \mathrm{H}_{1}\right)-\mathrm{F}_{1} \mathrm{H}_{2}+\mathrm{F}_{2} \mathrm{H}_{1}\right)
\end{array}\right],
\end{aligned}
$$

where

$$
\begin{aligned}
\Delta=- & \mathrm{W}_{1}\left(\mathrm{~F}_{2}\left(\mathrm{G}_{3} \mathrm{H}_{4}-\mathrm{G}_{4} \mathrm{H}_{3}\right)+\mathrm{F}_{3}\left(\mathrm{G}_{4} \mathrm{H}_{2}-\mathrm{G}_{2} \mathrm{H}_{4}\right)+\mathrm{F}_{4}\left(\mathrm{G}_{2} \mathrm{H}_{3}-\mathrm{G}_{3} \mathrm{H}_{2}\right)\right)+ \\
& \mathrm{W}_{2}\left(\mathrm{~F}_{1}\left(\mathrm{~W}_{3} \mathrm{H}_{4}-\mathrm{G}_{4} \mathrm{H}_{3}\right)+\mathrm{F}_{3}\left(\mathrm{G}_{4} \mathrm{H}_{1}-\mathrm{G}_{1} \mathrm{H}_{4}\right)+\mathrm{F}_{4}\left(\mathrm{G}_{1} \mathrm{H}_{3}-\mathrm{G}_{3} \mathrm{H}_{1}\right)\right)- \\
& \mathrm{W}_{3}\left(\mathrm{~F}_{1}\left(\mathrm{G}_{2} \mathrm{H}_{4}-\mathrm{G}_{4} \mathrm{H}_{2}\right)+\mathrm{F}_{2}\left(\mathrm{G}_{4} \mathrm{H}_{1}-\mathrm{G}_{1} \mathrm{H}_{4}\right)+\mathrm{F}_{4}\left(\mathrm{G}_{1} \mathrm{H}_{2}-\mathrm{G}_{2} \mathrm{H}_{1}\right)\right)+ \\
& \mathrm{W}_{4}\left(\mathrm{~F}_{1}\left(\mathrm{G}_{2} \mathrm{H}_{3}-\mathrm{G}_{3} \mathrm{H}_{2}\right)+\mathrm{F}_{2}\left(\mathrm{G}_{3} \mathrm{H}_{1}-\mathrm{G}_{1} \mathrm{H}_{3}\right)+\mathrm{F}_{3}\left(\mathrm{G}_{1} \mathrm{H}_{2}-\mathrm{G}_{2} \mathrm{H}_{1}\right)\right)
\end{aligned}
$$

Those complete the solution in the Laplace transform domain.

\section{NUMERICAL INVERSION OF THE LAPLACE TRANSFORMS}

In order to invert the Laplace transforms, we adopt a numerical inversion method based on a Fourier series expansion [27].

By this method the inverse $f(t)$ of the Laplace transform $\bar{f}(s)$ is approximated by

$$
\mathrm{f}(\mathrm{t})=\frac{\mathrm{e}^{\mathrm{ct}}}{\mathrm{t}_{1}}\left[\frac{1}{2} \overline{\mathrm{f}}(\mathrm{c})+\mathrm{R} 1 \sum_{\mathrm{k}=1}^{\mathrm{N}} \overline{\mathrm{f}}\left(\mathrm{c}+\frac{\mathrm{ik} \pi}{\mathrm{t}_{1}}\right) \exp \left(\frac{\mathrm{ik} \pi \mathrm{t}}{\mathrm{t}_{1}}\right)\right], \quad 0<\mathrm{t}_{1}<2 \mathrm{t},
$$

Where $\mathrm{N}$ is a sufficiently large integer representing the number of terms in the truncated Fourier series, chosen such that

$$
\exp (\mathrm{ct}) \mathrm{R} 1\left[\overline{\mathrm{f}}\left(\mathrm{c}+\frac{\mathrm{iN} \pi}{\mathrm{t}_{1}}\right) \exp \left(\frac{\mathrm{iN \pi t}}{\mathrm{t}_{1}}\right)\right] \leq \varepsilon_{1}
$$

where $\varepsilon_{1}$ is a prescribed small positive number that corresponds to the degree of accuracy required. The parameter $\mathrm{c}$ is a positive free parameter that must be greater than the real part of all the singularities of $\bar{f}(\mathrm{~s})$. The optimal choice of $\mathrm{c}$ was obtained according to the criteria described 
in [27].

\section{NUMERICAL RESULTS AND DISCUSSION}

The Ferrari's method has been constructed by using the FORTRAN program to solve equation (41). The material properties of asphaltic material saturated by water have been taken as follow [28], [29]:

$$
\begin{aligned}
& \mathrm{T}_{0}=27^{\circ} \mathrm{C}, \quad \mathrm{Q}=0.4853 \times 10^{11} \text { dyne. } \mathrm{cm}^{-2}, \quad \mathrm{R}=0.0362 \times 10^{11} \text { dyne. } \mathrm{cm}^{-2}, \lambda=0.2160 \times 10^{11} \text { dyne. } \mathrm{cm}^{-2} \\
& \mu=0.0926 \times 10^{11} \text { dyne.cm }{ }^{-2}, \alpha^{\mathrm{s}}=2.16 \times 10^{-5}{ }^{\circ} \mathrm{C}^{-1}, \rho^{\mathrm{s}^{*}}=2.35 \mathrm{gm} . \mathrm{cm}^{-3}, \rho_{11}=0.002 \mathrm{gm} . \mathrm{cm}^{-3} \\
& \mathrm{k}^{\mathrm{s}^{*}}=0.8 \mathrm{~W} \mathrm{~m}^{-1}{ }^{\mathrm{o}} \mathrm{k}^{-1}, \mathrm{C}_{\mathrm{E}}^{\mathrm{s}}=800 \mathrm{~J} \mathrm{~kg}^{-1} \cdot{ }^{\circ} \mathrm{C}^{-1}, \tau_{\mathrm{o}}^{\mathrm{s}}=0.02 \mathrm{~s}, \mathrm{k}=0.001 \mathrm{~W} \mathrm{~m}^{-1}{ }^{\mathrm{o}} \mathrm{k}^{-1} \\
& \alpha^{\mathrm{f}}=\alpha^{\mathrm{sf}}=\alpha^{\mathrm{fs}}=0.0001{ }^{\circ} \mathrm{C}^{-1}, \rho^{\mathrm{f} *}=0.82 \mathrm{gm} . \mathrm{cm}^{-3}, \mathrm{k}^{\mathrm{f}^{*}}=0.3 \mathrm{~W} \mathrm{~m}^{-1}{ }^{\circ} \mathrm{k}^{-1}, \mathrm{C}_{\mathrm{E}}^{\mathrm{f}}=1.9 \mathrm{cal} \cdot \mathrm{gm}^{-1} \cdot{ }^{\circ} \mathrm{C}^{-1} \text {, } \\
& \tau_{\mathrm{o}}^{\mathrm{f}}=0.00001 \mathrm{~s},
\end{aligned}
$$

We will take the non-dimensional $\mathrm{x}$ variable to be in interval $0 \leq \mathrm{x} \leq 1$ and all the results will be calculated at the same instance $t=0.1$ for two different values of the porosity $\beta$ of the material when $\beta=0.25$ and $\beta=0.35$.

The temperature, the stress, the strain and the displacement for the solid and the liquid have been shown in figures 1-8 respectively. We can see that, the value of the porosity has a significant effect on all the studied fields.

Figure 1 shows the temperature increment distribution of the solid with two different values of the porosity; $\beta=0.25$ and $\beta=0.35$. It shows that the porosity parameter has a significant effect.

Figure 2 shows the temperature increment distribution of the liquid with two different values of the porosity; $\beta=0.25$ and $\beta=0.35$. We can see that the porosity parameter has a significant effect where the liquid temperature increases when the porosity increases.

Figure 3 shows the stress distribution of the solid with two different values of the porosity; $\beta=0.25$ and $\beta=0.35$. We can see that the porosity parameter has a significant effect where the absolute value of the stress acts on the solid increases when the porosity increases.

Figure 4 shows the stress distribution of the liquid with two different values of the porosity; $\beta=0.25$ and $\beta=0.35$. We can see that the porosity parameter has a significant effect where the absolute value of the stress acts on the liquid increases when the porosity increases for wide range of $\mathrm{x}$.

Figures 5-8, show that the porosity parameter has significant effects on the deformation and the displacement for both medium solid and liquid. The absolute value of the peak points (sharp points) increase when the value of the porosity parameter increases for the both medium solid and water. 
International Journal on Soft Computing (IJSC) Vol.8, No. 1, February 2017

\section{CONCLUSION}

In studying a mathematical model of generalized porothermoelasticity with one relaxation time for poroelastic half-space saturated with fluid in the context of Youssef model we found that:

1- The porosity parameter of the poroelastic material has significant effects on the temperature, the stress, the deformation and the displacement distributions for the both medium the solid and the liquid.

2- Youssef model of porothermoelasticity with one relaxation time introduce finite speed of thermal wave propagation which agree with realistic physical behavior for the solid and the liquid.

\section{REFERENCES}

[1] C. Pecker, and H. Deresiewiez, Thermal Effects on Wave in Liquid-Filled Porous Media, J. Acta Mech. 16, 45-64, (1973).

[2] Biot, M. A. General theory of three-dimensional consolidation. J. Appl. Phys., 12, 155-164, (1941).

[3] Biot, M. A., Theory of propagation of elastic waves in a fluid-saturated porous solid. I. Lowfrequency range. J. Acoust. Soc. Am., 28, 168-178, (1956).

[4] Gassmann, F. Uber die elastizitat poroser medien. Veirteljahrsschrift der Naturforschenden Gesellschaft in Zzirich, 96, 1-23, (1951).

[5] Biot, M. A., and Willis, D. G. The elastic coefficients of the theory of consolidation. J. Appl. Mech., 24, 594-601, (1957).

[6] Biot, M. A. Mechanics of deformation and acoustic propagation in porous media. J. Appl. Phys., 33, 1482-1498, (1962).

[7] Deresiewicz, H. and Skalak, R., On uniqueness in dynamic poroelasticity, Bull. Seismol. Soc. Am. 53, 783-788, (1963).

[8] Mandl, G., Change in skeletal volume of a fluid-filled porous body under stress, J. Mech. Phys. Solids, 12, 299-315, (1964).

[9] Nur, A. and Byerlee, J. D. An exact effective stress law for elastic deformation of rock with fluids. J Geophys. Res., 76, 6414-6419, (1971).

[10] Brown, R. J. S. and Korringa, J. On the dependence of the elastic properties of a porous rock on the compressibility of the pore fluid. Geophysics, 40, 608-616, (1975).

[11] Rice, J. R.., and Cleary, M. P. Some basic, stress diffusion solutions for fluid-saturated elastic porous media with compressible constituents. Rev. Geophys. Space Phys., 14, 227-24, (1976).

[12] Burridge, R., and Keller, J. B. Poroelasticit, y equations derived from microstructure. J. Acoust. Soc. Am. 70, 1140-1146, (1981).

[13] Zimmerman, R. W., Somerton, W. H., and King, M. S. Compressibility of porous rocks. J. Geophys. Res., 91, 12765-12777, (1986).

[14] Zimmerman, R. W., Myer, L. R., and Cook, N. G. W. Grain and void compression in fractured and porous rock. Int. J. Rock Mech. Min. Sci. and Geomech. Abst., 31, 179-184, (1994).

[15] Berryman, J. G., and Milton, G. W. Exact results for generalized Gassmann's equations in composite porous media with two constituents. Geophysics, 56, 1950-1960, (1991).

[16] Thompson, M., and Willis, J.R.. A reformation of the equations of anisotropic poroelasticity. J. Appl. Mech., 58, 612-616, (1991).

[17] Pride, S. R., Gangi, A. F., and Morgan, F. D. Deriving the equations of motion for porous isotropic media. J. Acoust,. Soc. Am., 92, 3278-3290, (1992).

[18] Berryman, J. G., and Wang, H. F. The elastic coefficients of double-porosity models for fluid transport in jointed rock. J. Geophys. Res., 100, 24611-24627, (1995).

[19] Tuncay, K., and Corapcioglu, M. Y. Effective stress principle for saturated fractured porous media. Water Resources Res., 31, 3103-3106, (1995).

[20] Cheng, A. H. D., Integral equation for Poroelasticity in Frequency Domain with BEM solution, J. Eng. Mech. 117, No. 5, pp. 1136-1157, (1991).

[21] Charlez, P.A. and Heugas, O., Measurement of thermoporoelastic properties of rocks: theory and applications, Ed. J.A. Hudson, pp. 42-46, (1992). 
International Journal on Soft Computing (IJSC) Vol.8, No. 1, February 2017

[22] Abousleiman, Y. and Cui, L.,"Poroelastic Solutions in Transversely Isotropic Media for Wellbore and Cylinder," International Journal of Solids Structures., 35, Nos 34-35, pp. 4905-4903, (1998).

[23] Ghassemi, A., Diek, A., Porothermoelasticity for swelling shales. J. Petroleum Sci. \& Engineeing, 34, pp.123-135, (2002).

[24] S. R. Tod, An anisotropic fractured poroelastic effective medium theory, Geophys. J. Int. 155, 1006-1020, (2003).

[25] H. Lord and Y. Shulman, A Generalized Dynamical Theory of Thermoelasticity, J. Mech. Phys. Solid, vol. 15, pp. 299-309, (1967).

[26] Youssef, H. M., Theory of generalized porothermoelasticity, International Journal of Rock Mechanics and Mining Sciences, Vol. 44, pp.222-227, (2007).

[27] G. Honig and U. Hirdes, A method for the numerical inversion of Laplace Transform, J. Comp. Appl. Math., vol. 10, p. 113-132, (1984).

[28] Gan-bin Liu · Shi-rong Ding · Rong-hua Ye · Xiao-hu Liu, Relaxation Effects of a Saturated Porous Media Using the Two-Dimensional Generalized Thermoelastic Theory, J. Transp Porous Med, DOI 10.1007/s11242-010-9621-9, (2010).

[29] Yang Zhong - Litao Geng, Thermal stresses of asphalt pavement under dependence of material characteristics on reference temperature, J. Mech. Time-Depend Mater., vol.13, p. 81-91, (2009).

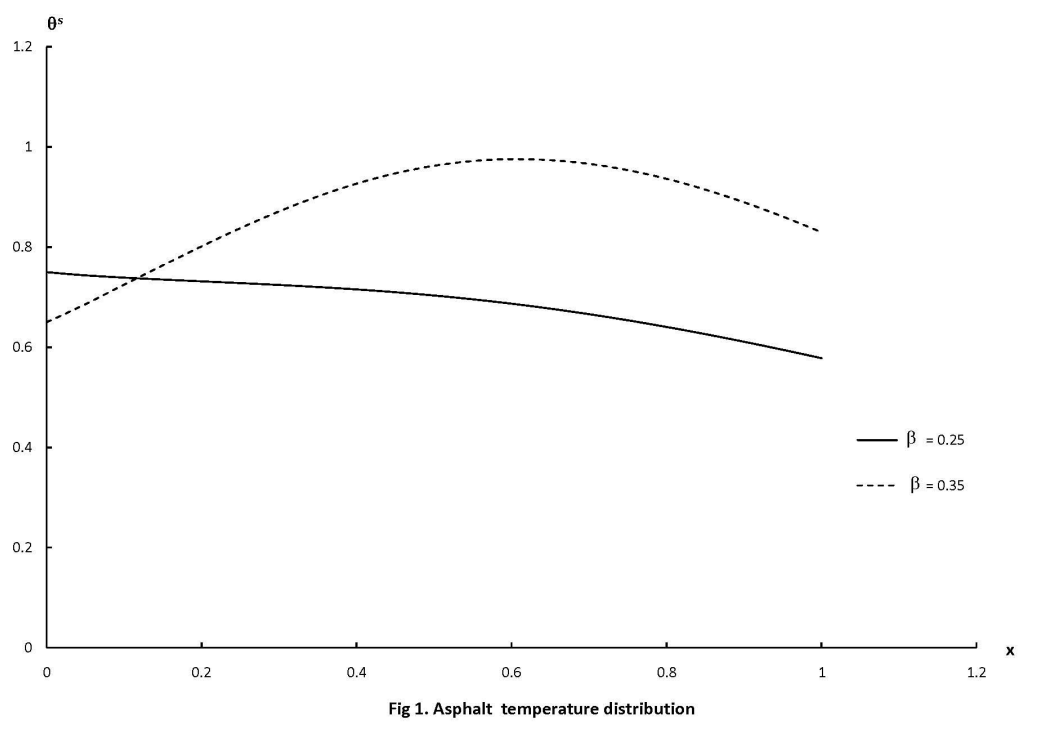


International Journal on Soft Computing (IJSC) Vol.8, No. 1, February 2017
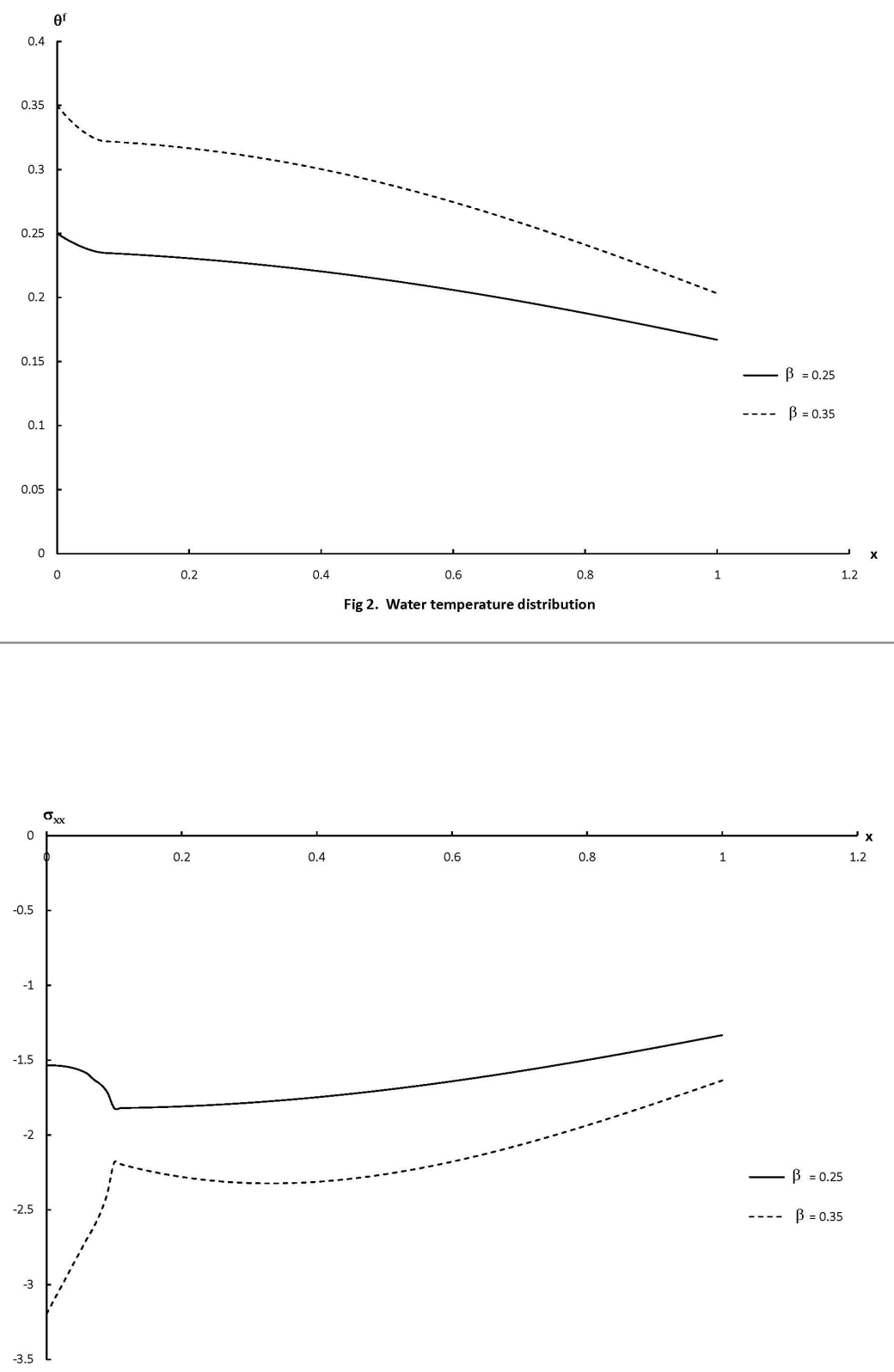

Fig 3. Asphalt stress distribution 
International Journal on Soft Computing (IJSC) Vol.8, No. 1, February 2017
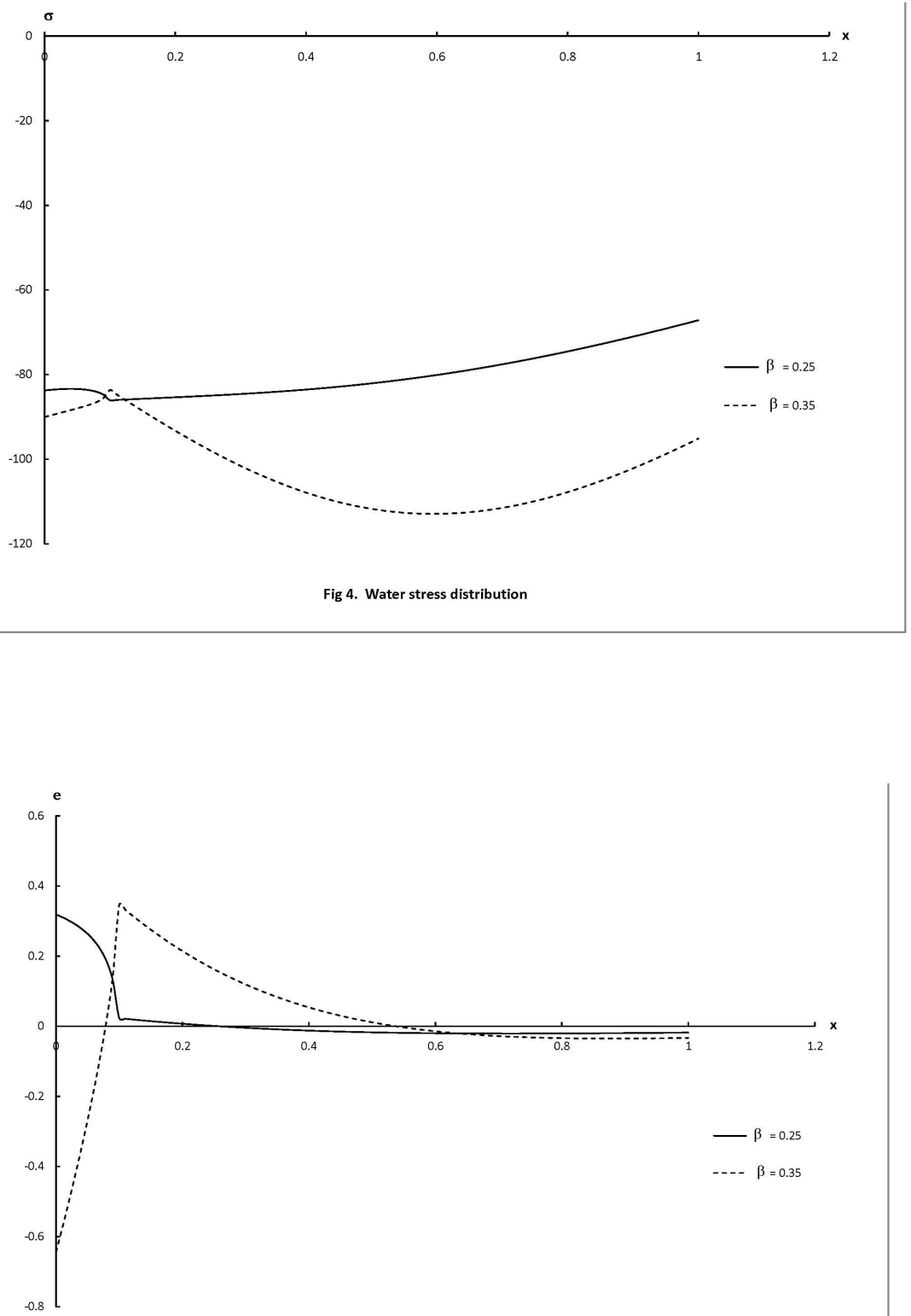

Fig 5. Asphalt strain distribution 
International Journal on Soft Computing (IJSC) Vol.8, No. 1, February 2017

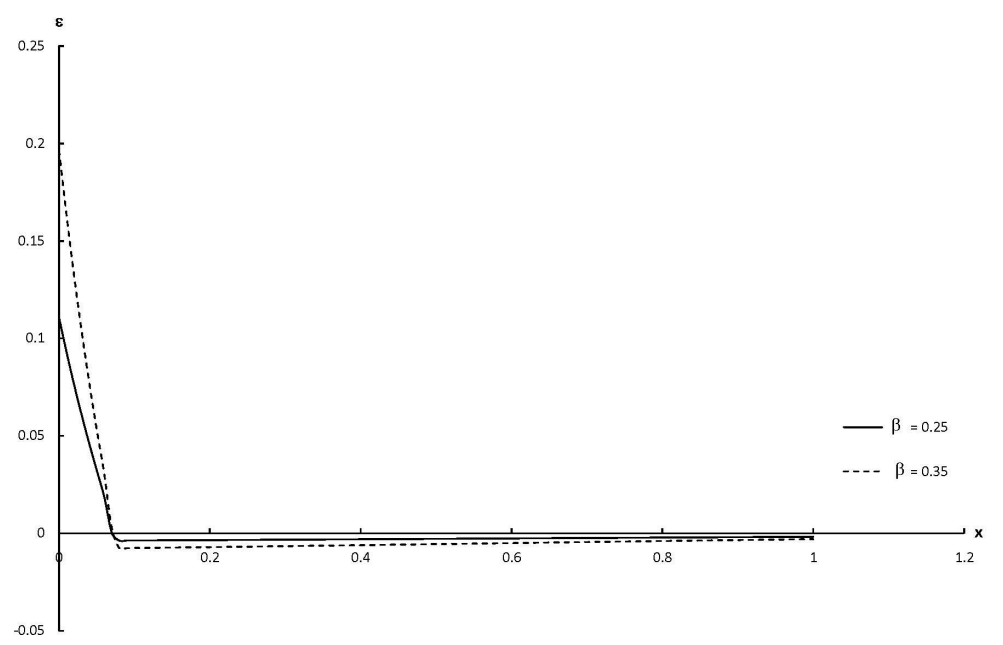

Fig 6. Water strain distribution

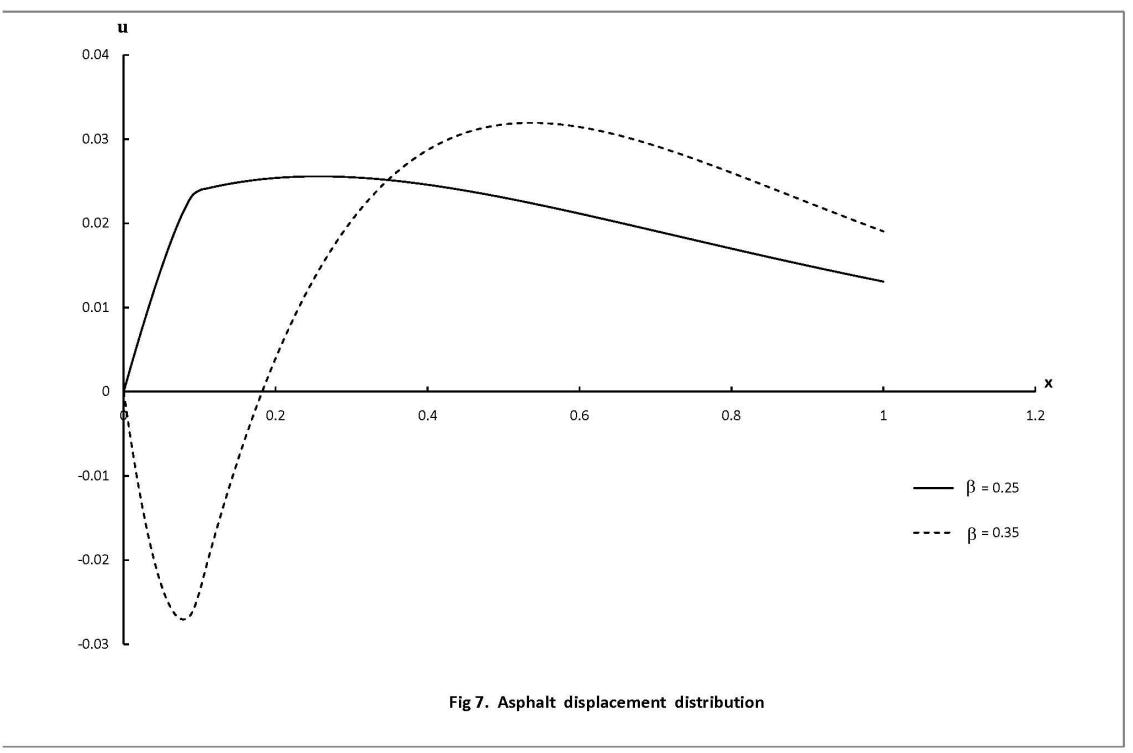


International Journal on Soft Computing (IJSC) Vol.8, No. 1, February 2017

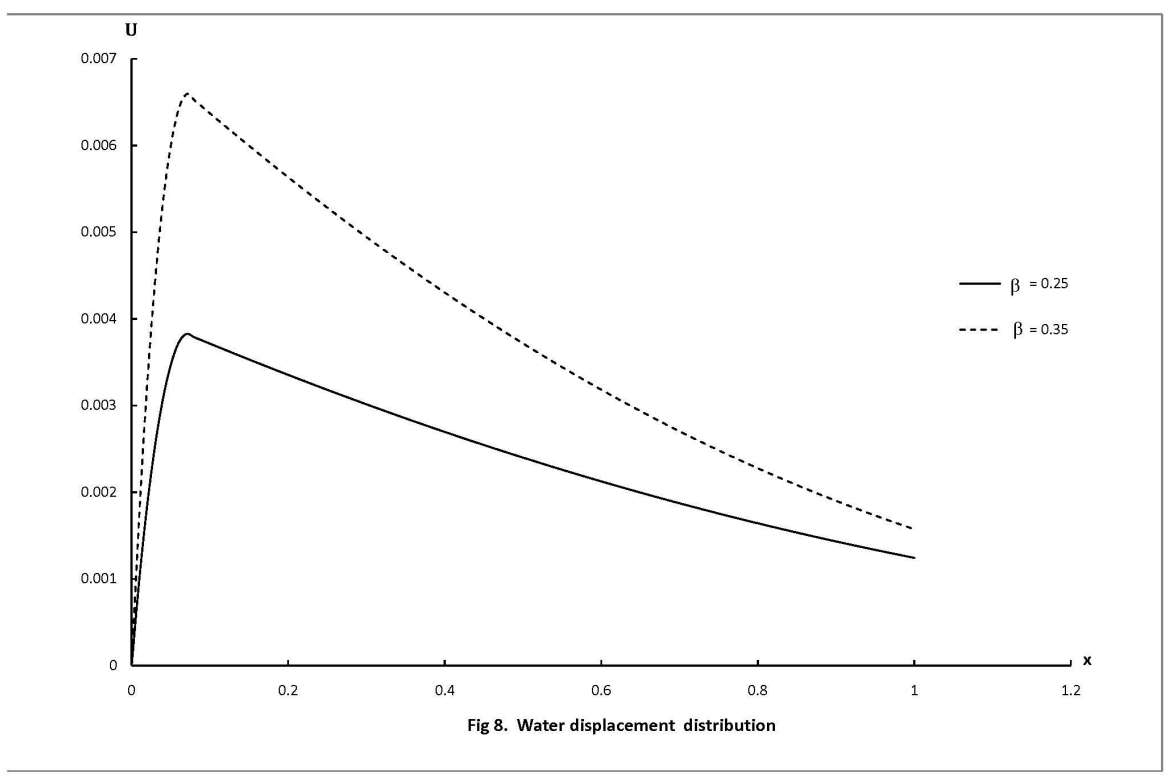

